The Astrophysical Journal, 260:L45-L47, 1982 September 15

(C) 1982. The American Astronomical Society. All rights reserved. Printed in U.S.A.

\title{
INTERSTELLAR LINES IN THE SPECTRA OF THE GLOBULAR CLUSTERS AROUND NGC 5128
}

\author{
Judith G. CoHEN \\ Department of Astronomy, California Institute of Technology \\ Received 1982 March 22; accepted 1982 May 27
}

\begin{abstract}
Three of the brightest globular clusters associated with the peculiar radio galaxy NGC 5128 have been observed spectroscopically. In addition to interstellar $\mathrm{Ca}$ II lines from our own Galaxy, two of the three show interstellar $\mathrm{Ca}$ II lines arising in NGC 5128. The radial velocities are not consistent with gas which shares the motion of the elliptical galaxy. In one case, the gas is possibly associated with the rotating inclined disk of gas seen close to the nucleus. The interstellar clouds are between 12 and $30 \mathrm{kpc}$ from the nucleus of NGC 5128, depending on whether they are in an extension of the rotating disk and how the disk is warped.
\end{abstract}

Subject headings: clusters: globular — galaxies: individual - interstellar: matter

\section{OBSERVATIONS}

Graham and Phillips (1980) discovered the first globular cluster associated with the peculiar radio galaxy NGC 5128 (Centaurus A). Several additional globular clusters were found by van den Bergh, Hesser, and Harris (1981, subsequently VHH). We obtained sky-subtracted spectra of three of the brightest of these clusters with the intensified Reticon detector constructed by S. Shectman on the $2.2 \mathrm{~m}$ du Pont telescope of the Las Campanas Observatory on 1981 May 25. The 3 pixel resolution (FWHM) was $3 \AA$ and the integration time was 1 hour per object. At $3950 \AA$, the count rate for the NGC 5128 globular clusters through a $2^{\prime \prime} \times 2^{\prime \prime}$ slit was about twice that of the sky.

Figure 1 shows the region $3920-4020 \AA$ in the smoothed spectra of each of the three globular clusters. A Gaussian convolution whose width was 5 pixels was used. The scatter in wavelength of individual $\mathrm{He}$ or Ar lines about the fourth-order polynomial fit to the wavelengths of 22 comparison lines was $\pm 0.5 \AA\left(40 \mathrm{~km} \mathrm{~s}^{-1}\right)$. G3 (globular number 3 on the chart of VHH) has in its spectrum a strong, narrow absorption at $3966.6 \AA$, which undoubtedly arises from interstellar absorption in our Galaxy. The galactic latitude of NGC 5128 is $19^{\circ}$, and the expected radial velocity for gas at the point closest to the galactic center along the line of sight is about $-40 \mathrm{~km} \mathrm{~s}^{-1}$. There are wider $\mathrm{H}$ and $\mathrm{K}$ absorption lines at $v_{r}=565 \mathrm{~km} \mathrm{~s}^{-1}$, which presumably are those of the globular cluster itself. VHH determined a radial velocity of $588 \mathrm{~km} \mathrm{~s}^{-1}$ for this cluster. Additional sharp absorptions are detected at the positions of $\mathrm{H}$ and $\mathrm{K}$ corresponding to $v_{r}=1295 \mathrm{~km} \mathrm{~s}^{-1}$ whose strengths are comparable to the interstellar Ca II absorption of our own Galaxy. With the aid of hindsight, all three of these features can be seen in Figure 2 of VHH.
In this spectral region, G5 has two wide absorption lines, presumably those of the globular cluster itself, which when interpreted as $\mathrm{H}$ and $\mathrm{H} \varepsilon$ give $v_{r}=530$ $\mathrm{km} \mathrm{s}^{-1}$, while for this globular cluster, VHH found $502 \mathrm{~km} \mathrm{~s}^{-1}$. There are additional sharp components present corresponding to interstellar $\mathrm{H}$ and $\mathrm{K}$ at a $v_{r}=890 \mathrm{~km} \mathrm{~s}^{-1}$. The absorption due to our own Galaxy, if present, is less than half that shown by G3. Interstellar absorption from galactic gas which is strongly variable over such a small angular scale is also seen in spectra of members of the globular cluster M22 (Cohen 1981).

G7 has very strong, broad $\mathrm{H}$ and $\mathrm{K}$ absorption at $v_{r}=550 \mathrm{~km} \mathrm{~s}^{-1}$. (VHH determined the radial velocity of $\mathrm{G} 7$ to be $571 \mathrm{~km} \mathrm{~s}^{-1}$.) Any absorption from the Milky Way must be less than half that shown by G3. No definite interstellar absorption was detected at any velocity.

Although these interstellar absorption features in NGC 5128 are close to the limit of credibility for the present data, the absorption detected in G5 is undoubtedly real as both $\mathrm{H}$ and $\mathrm{K}$ are clearly present with an appropriate ratio of equivalent widths. The highvelocity component claimed for $\mathrm{G} 3$ is more dubious and needs confirmation, which is apparently provided by the spectra of VHH. The intensified reticon detector is, as noted by Shectman and Hiltner (1976), at low count rates limited only by Poisson statistics, at least until a $2 \%$ noise-to-signal ratio is achieved.

\section{LOCATION OF THE ABSORBING GAS}

Graham (1979) has studied the velocity field of NGC 5128 , whose systemic velocity is $548 \mathrm{~km} \mathrm{~s}^{-1}$. He finds that the main body of this peculiar galaxy in many respects resembles a normal elliptical galaxy, and no 


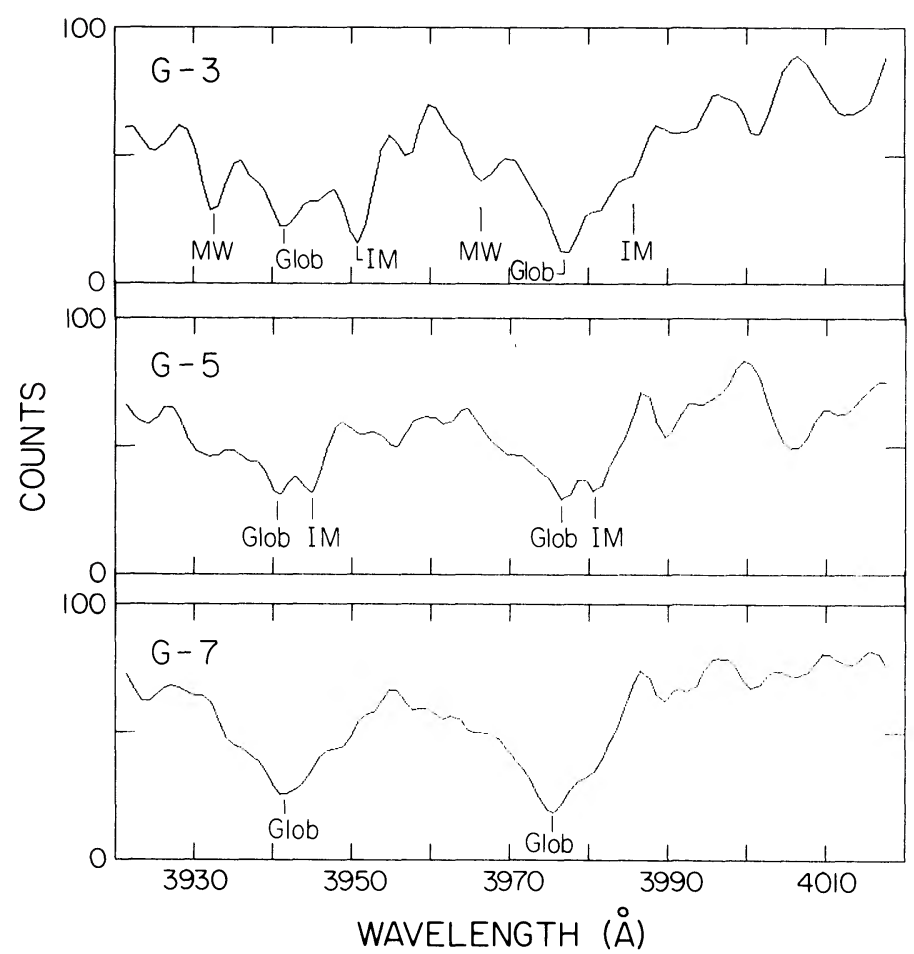

Fig. 1.- Smoothed spectra of three globular clusters associated with NGC 5128 are shown for the wavelength interval $3920-4020 \AA$. The interstellar absorptions from our Galaxy are denoted by MW, those from high-velocity gas in NGC 5128 by IM, and the absorption in the integrated light of the globular cluster itself by Glob.

rotation was detected. There is also an inclined rotating disk of gas and stars whose axis of rotation lies along the major axis of the elliptical component. Other such cases of skewed disks of gas in elliptical galaxies have recently been discovered. For example, Raimond et al. (1981) found that the outer part of the $\mathrm{H}$ I gaseous ring in the elliptical galaxy NGC 4278 is not coplanar with the inner optical galaxy.

The radio emission seen in NGC 5128 is a double double-lobed structure; the inner double lobe is aligned with the major axis of the elliptical galaxy (Christiansen et al. 1977). Faint optical filaments and emission knots have been seen by Blanco et al. (1975) and by Peterson, Dickens, and Cannon (1975) just beyond the edge of the elliptical component along the direction of the northeast radio lobe. These features have no detectable motion with respect to the nucleus of the galaxy.

We assume NGC 5128 is at a distance of $5 \mathrm{Mpc}$. The distances projected onto the plane of the sky of G3, G5, and G7 from the nucleus of the galaxy are then 10, 12, and $12 \mathrm{kpc}$ respectively. We have detected two probable sets of interstellar Ca II lines arising within NGC 5128: the $1295 \mathrm{~km} \mathrm{~s}^{-1}$ component in G3 and the $890 \mathrm{~km} \mathrm{~s}^{-1}$ component in G5. As the elliptical shows no rotation, it is not possible for either of these interstellar clouds to be associated with the stellar component of the elliptical galaxy.
It seems more likely that the clouds are associated with the rotating disk of gas and young stars. The sign of the velocity is correct, in that the gas is receding from us in the position angles corresponding to G3 and G5. However, the maximum radial velocity within the rotating disk detected by Graham (1979), which was seen at the maximum distance from the nucleus observed ( $\left.3^{\prime}\right)$, was only $800 \mathrm{~km} \mathrm{~s}^{-1}$. If the disk is not badly warped and if its rotational velocity increases outward (as Rubin, Ford, and Thonnard 1980 have shown is the case for Sc spirals), the gas within the disk can give rise to the component seen in G5. The $1295 \mathrm{~km} \mathrm{~s}^{-1}$ component in G3 is receding too rapidly to arise from gas in the rotating disk and its origin remains obscure. Note that because the rotating disk is inclined at an angle of $73^{\circ}$ with respect to the plane of the sky (Graham 1979), the distance from the nucleus to the point where the line of sight to G3 or G5 intersects the plane of the disk must be at least $30 \mathrm{kpc}$.

Two major hypotheses have been advanced to explain the peculiarities of NGC 5128. The presence of two separate galaxies coincidentally along the same line of sight was originally suggested by Baade and Minkowski (1954). Graham (1979), Tubbs (1980), and others have advocated NGC 5128 as a case of a merger of two galaxies (or a galaxy plus a gas cloud). In contrast, van Albada, Kotanyi, and Schwarzchild (1982) suggest that 
the rotating ring of gas could be a permanent, stable dynamical feature in a triaxial stellar system. If the absorption from either G3 or G5 arises in the disk gas, the necessary large size of the disk, even if it is significantly warped, would strengthen the case for a galaxy merger.

\section{SUMMARY}

The discovery by VHH of a system of globular clusters surrounding NGC 5128 offers a unique opportunity to carry out a probe of the interstellar medium, its morphology and dynamics, in the outer part of this peculiar radio galaxy. Our detection of optical interstellar absorption lines in the spectra of two of these globular clusters has made a start toward this goal by demonstrating that gas possibly associated with the rotating disk exists far out in the halo of NGC 5128. Much more work remains to be done. The objects are faint, and further progress will be slow and difficult.

I am grateful to the Director of the Mount Wilson and Las Campanas Observatory for a generous allocation of observing time.

\section{REFERENCES}

Baade, W., and Minkowski, R. 1954, Ap. J., 119, 215.

Blanco, V. M., Graham, J. A., Lasher, B. M., and Osner, P. S. 1975, Ap. J.' (Letters), 198, L63.

Christiansen, W. N., Frater, R. H., Watkinson, A., O'Sullivan, J. D., Lockhard, I., and Goss, W. M., 1977, M.N.R.A.S., 181, 183.

Cohen, J. G. 1981, Ap. J., 247, 869.

Graham, J. A., 1979, Ap. J., 232, 60.

Graham, J. A., and Phillips, M. M., 1980, Ap. J. (Letters), 239, L37.

Peterson, B. A., Dickens, R. J., and Cannon, R. D., 1975, Proc. Astr. Soc. Australia, 2, 367.

Raimond, E., Faber, S. M., Gallagher, J. S., and Knapp, G. R. 1981, Ap. J., 246, 708

Rubin, V. C., Ford, W. K., and Thonnard, N. 1980, Ap. J., 238, 471.

Shectman, S. A., and Hiltner, W. A., 1976, Pub. A.S.P., 88, 960. Tubbs, A. D. 1980, Ap. J., 241, 969.

van Albada, T. S., Kotanyi, C. G., and Schwarzchild, M. 1982, M.N.R.A.S., 198, 303.

van den Bergh, S., Hesser, J. E., and Harris, G. L. 1981, A.J., 86, $24(\mathrm{VHH})$.

Judith G. CoHEN: 105-24, California Institute of Technology, Pasadena, CA 91125 